\title{
De novo transcriptome assembly reveals high transcriptional complexity in Pisum sativum axillary buds and shows rapid changes in expression of diurnally regulated genes
}

Stephanie C. Kerr, Federico Gaiti, Christine A. Beveridge and Milos Tanurdzic ${ }^{*}$

\begin{abstract}
Background: The decision for a bud to grow into a branch is a key regulatory process affecting plant architecture. In order to study molecular processes regulating axillary bud outgrowth in the model plant garden pea (Pisum sativum), we sequenced the axillary bud transcriptome and performed de novo transcriptome assembly.

Results: We assembled a pea axillary bud transcriptome into 81,774 transcripts comprised of 194,067 isoforms. This new pea transcriptome resource is both comprehensive and representative, as shown by comparison to other available pea sequence resources. Over half of the transcriptome could be annotated based on sequence homology to Arabidopsis thaliana proteins, while almost one quarter of the isoforms were identified as putative long non-coding RNAs (IncRNAs). This transcriptome will be useful in studies of pea buds because it includes genes expressed specifically in buds which are not represented in other transcriptome studies. We also investigated the impact of a short time collection series on gene expression. Differential gene expression analysis identified 142 transcripts changing within the short 170 min time frame that the buds were harvested within. Thirty-three of these transcripts are implicated in diurnal fluctuations in other flowering plants, while the remaining transcripts include 31 putative IncRNA. Further investigation of the differentially expressed transcripts found an enrichment of genes involved in post-transcriptional regulation, including RNA processing and modification, as well as genes involved in fatty acid biosynthesis and oxidative phosphorylation.

Conclusions: We have sequenced and assembled a high quality pea bud transcriptome containing both coding and non-coding RNA transcripts that will be useful for further studies into axillary bud outgrowth. Over the short sample collection time frame of just 170 min, we identified differentially expressed coding and non-coding RNA, some of which are implicated in diurnal regulation, highlighting the utility of our transcriptome resource in identifying gene expression changes and informing future experimental designs.
\end{abstract}

Keywords: Pisum sativum, RNA-seq, Transcriptome, Gene expression, Long non-coding RNA, Axillary buds, Diurnal

\footnotetext{
*Correspondence: m.tanurdzic@uq.edu.au

The University of Queensland, School of Biological Sciences, St Lucia, QLD 4072, Australia
} 


\section{Background}

Branching is a major determinant of shoot architecture in plants and highly influences the yield of agricultural crops. The formation of branches begins when small meristematic tissue develops in the axils of leaves to form axillary buds [1]. Once formed these buds usually remain in a state of suspended growth (dormant) until they receive a growth-triggering signal. These signals may be environmental, such as light, nutrients, and decapitation of the shoot tip, or endogenous, such as hormones and sugars [2]. The ability of a plant to respond to each of these signals determines its final shoot architecture.

There is still much debate and poor clarity on exactly how axillary buds are maintained dormant or promoted to grow out $[1,2]$. Multiple signaling pathways are known to regulate this process, but little is known about how these pathways are integrated and which signaling pathways predominate at which stages of development. For example, it has recently been shown that sugars are the likely initial trigger of bud outgrowth after decapitation of the shoot tip [3], but whether sugars also interact with hormone or other environmental signaling pathways is yet to be determined. Therefore, in order to better understand the genetic changes involved in bud outgrowth, we sequenced the first transcriptome of axillary buds from garden pea (Pisum sativum) plants. Garden peas have large buds separated by long internodes, making it easier to harvest bud tissue specifically. In addition, branching has been well studied in garden pea with many resources available, including branching mutants and curated transcriptome libraries [4].

This is the first of two gene expression studies of pea axillary buds using this new transcriptome resource. Here we report and describe the pea axillary bud transcriptome and identify a significant time-of-day experimental variable influencing gene expression during the sample collection time frame in the rms5-3 genetic background. These mutants are near-isogenic with the wild type cv. Torsdag widely used in developmental genetics and hormone studies, and produce very little endogenous strigolactone, a plant hormone which represses axillary bud outgrowth [2] therefore enabling inclusion of transcripts involved in active bud outgrowth. In addition to establishing a quality pea bud transcriptome, we identified gene expression changes in the growing axillary buds samples at different time windows over a $170 \mathrm{~min}$ time frame that are contributed by diurnal and circadian effects. These results have implications for future design of gene expression studies as most gene expression studies that utilize multiple treatments and replicates require the samples to be harvested over a period of time.

\section{Results and discussion}

Transcriptome sequencing and de novo garden pea axillary bud transcriptome assembly

We generated a pea axillary bud de novo transcriptome assembly from $\sim 55$ million 250 bp paired-end RNA-seq reads sequenced using Illumina MiSeq technology (Table 1; Additional file 1: Tables S1 and S2). The transcriptome comprised of reads from both mock and strigolactone treated buds of intact rms5-3 mutant plants treated for 1, 2, 4 and $6 \mathrm{~h}$, with each treatment time harvested over a $170 \mathrm{~min}$ time frame (Additional file 1: Table S2). The assembly produced 81,774 transcripts with 194,067 isoforms, an N50 isoform size of 2170 bp (Table 1) and a transcriptome size of $57 \mathrm{Mb}$ (Table 2). The isoform length distribution is shown in Additional file 1: Figure S1. The high number of assembled transcripts likely reflects the complex nature of a growing axillary bud.

\section{Assessment of the transcriptome assembly}

A number of criteria were used to determine the redundancy and quality of our pea bud transcriptome assembly, including comparisons to published transcriptomes from pea and other plant species, and alignment to available pea gene sequences.

\section{The pea axillary bud transcriptome redundancy and comparison to other pea gene sequences}

Firstly, we tested within-assembly redundancy by comparing the assembled isoforms to all other isoforms using BLASTN $\left(1 \mathrm{E}^{-03}\right)$ and a minimum hit coverage of $80 \%$ (Additional file 1: Table S3). Most of the matches were between isoforms from the same transcript, with only six matches between different transcripts. This suggests a very low redundancy of transcripts in our assembly.

As a number of pea transcriptomes have previously been published [5-8], we compared them with our pea bud transcriptome. Over $90 \%$ of the pea transcripts generated

Table 1 Summary of de novo pea axillary bud transcriptome statistics

\begin{tabular}{ll}
\hline Statistic & Pea bud transcriptome \\
\hline \# contigs & 81,774 \\
\# isoforms & 194,067 \\
Shortest isoform (bp) & 201 \\
Longest isoform (bp) & 17,155 \\
\# large isoforms (>1000 bp) & 108,011 \\
N50 isoforms (bp) & 2170 \\
Ave. isoform length (bp) & 1285 \\
\# paired reads used in assembly & $\sim 55$ million \\
\# nucleotides used in assembly & $\sim 23 \mathrm{~Gb}$ \\
Reads mapped in pairs (\%) & $58.67 \%$ \\
Individual reads mapped (\%) & $90.94 \%$ \\
\hline
\end{tabular}


Table 2 Estimated size of different Pisum sativum and other plant species transcriptomes

\begin{tabular}{lll}
\hline Species & Source & $\begin{array}{l}\text { Estimated transcriptome } \\
\text { size (Mb) }\end{array}$ \\
\hline Pisum sativum bud & - & $57^{\mathrm{a}}$ \\
Pisum sativum bud & - & $249^{\mathrm{b}}$ \\
Pisum sativum & {$[7]$} & 37 \\
Pisum sativum & {$[6]$} & 10 \\
Pisum sativum & {$[8]$} & 59 \\
Pisum sativum (Kaspa) & {$[5]$} & $81^{\mathrm{b}}$ \\
Pisum sativum (Parafield) & {$[5]$} & $72^{\mathrm{b}}$ \\
Medicago truncatula & Mt4.0v1 & $67^{\mathrm{c}}$ \\
Solanum lycopersicum & ITAG1 & $38^{\mathrm{c}}$ \\
Arabidopsis thaliana & TAIR10 & $66^{\mathrm{c}}$
\end{tabular}

${ }^{\text {a }}$ reflects the transcriptome size estimated using the longest isoform per contig ${ }^{b}$ reflects the transcriptome size estimated using all isoforms

cbased on whole genome reference

using earlier 454 sequencing technology [6-8] are contained within our transcriptome assembly (Table 3 ). In line with this, our transcriptome contained significantly more transcripts than the other transcriptomes (Table 3). These results are likely due to the increased sequence read length and depth used in our transcriptome sequencing, resulting in a more comprehensive transcriptome assembly and discovery of novel pea genes. This is further corroborated by comparisons to the more recent pea transcriptomes of the Kaspa and Parafield genotypes [5] that used similar Illumina sequencing technology to ours. In these cases, we found that $\sim 75 \%$ of our isoforms are present in the Kaspa and Parafield transcriptomes, while $\sim 78 \%$ of the Kaspa and Parafield transcripts were present in our transcriptome (Table 3). It is worth noting that all of the published pea transcriptomes comprise a number of different plant tissues while ours is restricted to the axillary bud. This may imply that many of the differences in overlap are the result of true biological differences between tissues, highlighting the complexity of the transcriptome of the developing axillary bud.
We also found that approximately $80 \%$ of pea protein sequences available in the Swiss-Prot and UniProtKB databases had a matching transcript in the transcriptome, using BLASTX $\left(1 \mathrm{E}^{-10}\right)$ with a minimum hit coverage of 80\% (Table 4). Therefore, our comprehensive de novo transcripts assembly has allowed us to generate a representative catalog of genes expressed in a pea bud.

\section{Inter-specific sequence comparison}

In the absence of garden pea reference genome sequences, we used the coding sequences (CDS) annotated in the whole genome assembly of the closest available relative, Medicago truncatula (Mt4.0v1 [9]), to annotate the axillary bud transcriptome isoforms using TBLASTX $\left(1 \mathrm{E}^{-10}\right)$. Over $60 \%$ of the pea isoforms matched to a Medicago CDS, while $\sim 77 \%$ of the Medicago CDS matched a pea isoform (Table 3). The discrepancy between the pairwise TBLASTX searches is likely explained by a combined effect of pea-specific and noncoding transcripts which is especially likely given the almost 9-fold increase in the garden pea genome size relative to Medicago [4], as well as alternatively spliced isoforms or potential mis-assemblies.

Furthermore, we compared our transcriptome to a list of 387 Ultra Conserved Orthologs (UCOs). These are single copy genes conserved across eukaryotes, specifically Arabidopsis thaliana, humans, mice, yeast, fruit flies and Caenorhabditis elegans [10]. Using BLASTX $\left(1 \mathrm{E}^{-10}\right)$ and a minimum $75 \%$ sequence coverage, we found $81 \%$ of the UCO sequences in our pea bud transcriptome (Table 4).

\section{Comparison to single copy gene sequences from pea}

We used an arbitrarily selected set of ten previously sequenced single copy pea genes [11-19] to determine how well each transcript has been assembled. BLASTX $\left(1 \mathrm{E}^{-100}\right)$ was used to compare these ten reference sequences against the pea bud transcriptome to determine how many copies of each gene was present in the transcriptome, and how well each transcript was assembled. Only two of the ten reference sequences, PsFed-1 and PSRMS5, had more than one copy in the transcriptome

Table 3 BLAST comparisons (E-value $1 \mathrm{E}^{-10}$ ) between the pea bud isoforms as the query and Pisum sativum contigs [5-8] or Medicago CDS and proteins as the reference

\begin{tabular}{llll}
\hline Reference & Analysis & \# reference matches & \# pea bud isoform matches \\
\hline Pea (Franssen) contigs & BLASTN & $78,692(93 \%)$ & $109,619(56 \%)$ \\
Pea (Kaur) contigs & BLASTN & $13,112(96 \%)$ & $46,411(24 \%)$ \\
Pea (Duarte) contigs & BLASTN & $65,732(96 \%)$ & $112,429(58 \%)$ \\
Pea (Kaspa) contigs & BLASTN & $98,613(79 \%)$ & $143,092(74 \%)$ \\
Pea (Parafield) contigs & BLASTN & $112,836(77 \%)$ & $147,661(76 \%)$ \\
Medicago (Mt4.0v1) CDS & TBLASTX & $48,203(77 \%)$ & $117,699(61 \%)$ \\
Medicago (Mt4.0v1) proteins & BLASTX & $47,385(76 \%)$ & $115,151(59 \%)$ \\
\hline
\end{tabular}


Table 4 BLASTX (E-value $1 \mathrm{E}^{-10}$ ) comparisons between the pea bud isoforms as the query and Ultra-Conserved Ortholog (UCO) protein sequences, Swiss-Prot pea or UniProtKB pea protein sequences as the reference

\begin{tabular}{llll}
\hline Reference & Min. subject coverage & \# reference matches & \# pea bud isoform matches \\
\hline UCO & $75 \%$ & $316(81 \%)$ & 320 \\
Swiss-Prot & $80 \%$ & $308(79 \%)$ & 812 \\
UniProtKB & $80 \%$ & $1210(80 \%)$ & 2289 \\
\hline
\end{tabular}

(Additional file 1: Table S4). Both of these transcripts had low read coverage over the reference transcript which could have impacted on the ability to properly assemble full-length transcripts (Additional file 2: Figures S5 and S11). Importantly, six of the reference sequences are matched by a single isoform covering the majority of the reference sequence (Additional file 1: Table S4; Additional file 2: Figures S2-S11), reflecting the high level of completeness of our assemblies. In support of this, eight of the assembled transcripts match at their $5^{\prime}$ end with the $5^{\prime}$ end of the reference sequences (Additional file 1: Figures S2-S11). As expected, transcript abundance affects this analysis, such that the transcripts of three pea genes with low abundance, PsRMS5, PSFed-1, and PSPETE, are found only partially assembled in the pea axillary bud transcriptome.

\section{Annotation}

In order to predict the putative function of the pea bud transcripts and their isoforms, BLASTX was used to align the isoforms, firstly with Arabidopsis thaliana proteins (1E $\left.{ }^{-10}\right)$, and secondly with all proteins from the NCBI nonredundant $(\mathrm{nr})$ sequence database $\left(1 \mathrm{E}^{-10}\right)$. This led to 55 and $61 \%$ of the isoforms, and 27 and $34 \%$ of the transcripts being annotated by protein sequence similarity, respectively (Additional file 3: Table S5; Additional file 4: Table S6).

When we compared the length of the isoforms annotated with the nr protein sequence database with the unannotated isoforms, we found that the average unannotated isoform was $472 \mathrm{bp}$ in length while the average annotated isoform was $\sim 4$ times longer, with an average length of
1805 bp (Fig. 1a). To account for the fact that some of the isoforms may contain untranslated regions, we also determined the open-reading frame (ORF) sizes of the isoforms. The average ORF size was $65 \mathrm{bp}$ for the unannotated isoforms and $320 \mathrm{bp}$ for the annotated isoforms, a similar fold-change to the difference in overall length between the two types of isoforms. The unannotated isoforms are mostly quite short in length, which suggests either that they are incomplete assemblies and so cannot be matched adequately to their homologues in other species, or that they may be non-coding RNAs.

To identify putative long non-coding RNAs (lncRNAs) in the pea bud transcriptome, we used a custom lncRNA prediction computational pipeline which took into account four core filtering criteria: 1) similarities to known protein sequences and protein domains, 2) the presence of signal peptides, 3) isoform length (>200 nt), and 4) open reading frame size $(\mathrm{ORF}<50$ aa). Using this approach, we found 47,322 putative IncRNAs (Additional file 5: Table S7), with a median length of $293 \mathrm{nt}$ and a range from 201 to 2781 nt (Additional file 1: Figure S12). Two-thirds of the unannotated isoforms were identified as putative lncRNAs (Fig. 1b); conversely, none of the annotated isoforms were putative lncRNAs.

The large number of putative lncRNAs in pea is at the higher end of the range of the number of lncRNAs predicted in other plants species with a genome sequence reference [20-22]. This could be the result of a much larger non-coding portion of the pea genome [23], and it would imply that some of the non-coding portion of the pea genome is transcribed. Indeed, sequence comparison
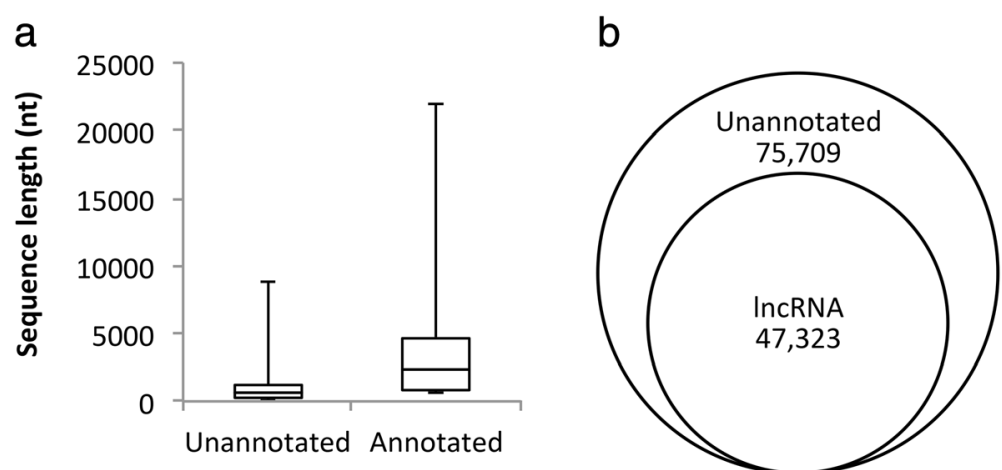

Fig. 1 a Comparison of the sequence length (nt) of unannotated and annotated isoforms from the de novo pea axillary bud assembly, and $\mathbf{b}$ the overlap between the number of unannotated isoforms and the number of IncRNA in the pea bud transcriptome 
between the predicted lncRNA and available pea repetitive DNA sequences [23] revealed that 12,034 (25\%) of the predicted lncRNA represent various types of repetitive portions of the pea genome, with transcripts of the LTR retrotransposons Ogre and Maximus, as well as unclassified repeats, representing $68 \%$ of all repetitive DNA transcripts (Additional file 1: Table S8). Furthermore, in the absence of a reference pea genome sequence, isoforms of lncRNA are not easily identifiable, potentially leading to inflated lncRNA counts. In addition, our RNA purification did not include a polyA+ selection step; therefore it is likely that some of the lncRNA may represent non-polyadenylated transcripts. Recently, novel non-polyadenylated transcripts have been detected in the model plants Arabidopsis and rice [24, 25]. These non-polyadenylated transcripts, known as intermediate sized ncRNAs (im-ncRNAs), are 50-300 nt in length, have low protein-coding potential, and do not show sequence similarity to any known ncRNA $[24,25]$.

Sequence comparisons to lncRNAs from Medicago [21] and Arabidopsis [20] were also made, identifying 1485 (6\%) and $114(0.3 \%)$ lncRNAs, respectively, conserved in pea (Additional file 5: Tables S9 and S10). As lncRNA are thought to be fast evolving and constrained by their secondary and tertiary structures $[26,27]$ and sometimes synteny [28], rather than just their sequence, detecting hundreds of sequence-conserved lncRNA provides a new resource for comparative analysis of lncRNA sequence and structure conservation in flowering plants.

The pea bud transcriptome was also annotated with the Rfam database of RNA families [29]. A total of 921 isoforms and 354 transcripts were annotated in this way (Additional file 5: Table S11), of which small nucleolar RNAs (snoRNAs) and microRNA (miRNA) precursors (Fig. 2) were the most highly represented RNA families. Interestingly, we identified 100 likely miRNA precursor transcripts

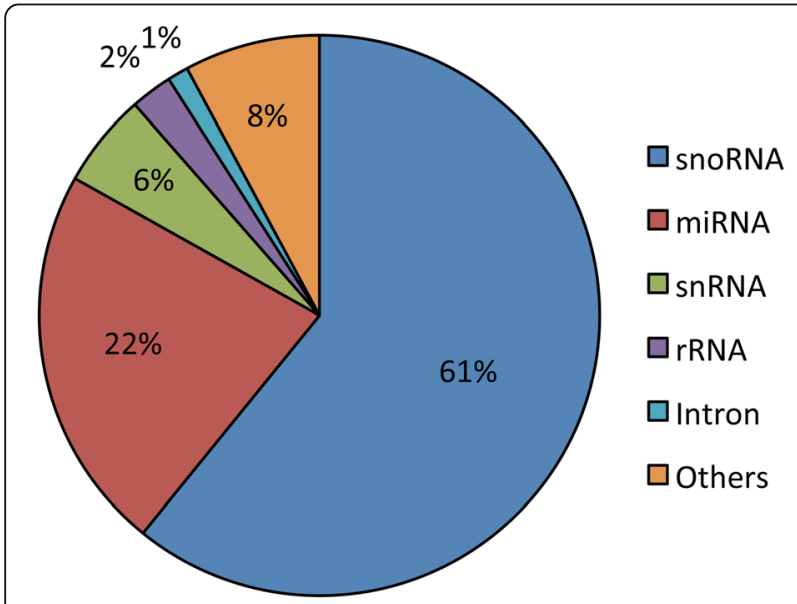

Fig. 2 Rfam non-coding RNA families represented in the pea bud transcriptome representing 31 miRNA gene families expressed in the pea axillary bud (Additional file 5: Table S11).

\section{Functional annotation of the pea axillary bud transcriptome}

We functionally annotated the pea axillary bud transcriptome using both gene ontology (GO) terms [30] and KEGG metabolic pathways [31]. We identified 140 KEGG pathways with at least one member of the pathway annotated in the pea bud transcriptome, and at least 20 KEGG pathways with more than $50 \%$ represented in the pea bud transcriptome (Additional file 5: Table S12). The KEGG pathways with the highest percentage of enzymes annotated in the transcriptome were carbon fixation in photosynthetic organisms with $21 / 25$ (82\%) enzymes annotated in the transcriptome, and glycolysis/gluconeogenesis with $25 / 31(80 \%)$ of the enzymes annotated in the transcriptome (Fig. 3; Additional file 1: Figure S13). This, along with the most highly represented GO categories which included numerous metabolic processes (Additional file 5: Tables S13-S15), supports the fact that active and diverse metabolic processes are occurring in the photosynthetically active buds as they are growing.

\section{Diurnally regulated transcripts in pea axillary buds}

To determine whether time of day had an effect on gene expression in axillary buds over the $170 \mathrm{~min}$ time frame of the collection of the samples, we used the RNA-seq data from our mock treated samples for differential gene expression analysis contrasting gene expression between the three collection time windows. In addition to identifying diurnally regulated genes in pea buds (based on expression and annotation), this analysis will inform future experimental design on axillary bud growth dynamics and gene expression studies. As the samples were collected in three groups between 1.30 and $4.30 \mathrm{pm}$ (Additional file 1: Table S16), we designated samples harvested between 1.30 and $2.10 \mathrm{pm}$ as time window 1 , samples harvested between 2.10 and $2.50 \mathrm{pm}$ as time window 2, and samples harvested between 3.50 and $4.30 \mathrm{pm}$ as time window 3 as described in the Methods section.

Pairwise comparisons of gene expression differences were made between each of the time windows (Table 5; Additional file 1: Tables S17 and S18), which identified a total of 142 unique differentially expressed (DE) transcripts. The time windows harvested closer together showed fewer DE transcripts between them, with no DE transcripts between time windows 1 and 2, and only 37 $\mathrm{DE}$ transcripts between time windows 2 and 3. The time windows that were the furthest apart, time windows 1 and 3, showed 124 DE transcripts. There was an overlap of 19 DE transcripts between the two analyses. We tested 14 randomly chosen DE transcripts by qRT-PCR; all 14 were identified as DE in our differential gene expression 
\% enzymes annotated in transcriptome

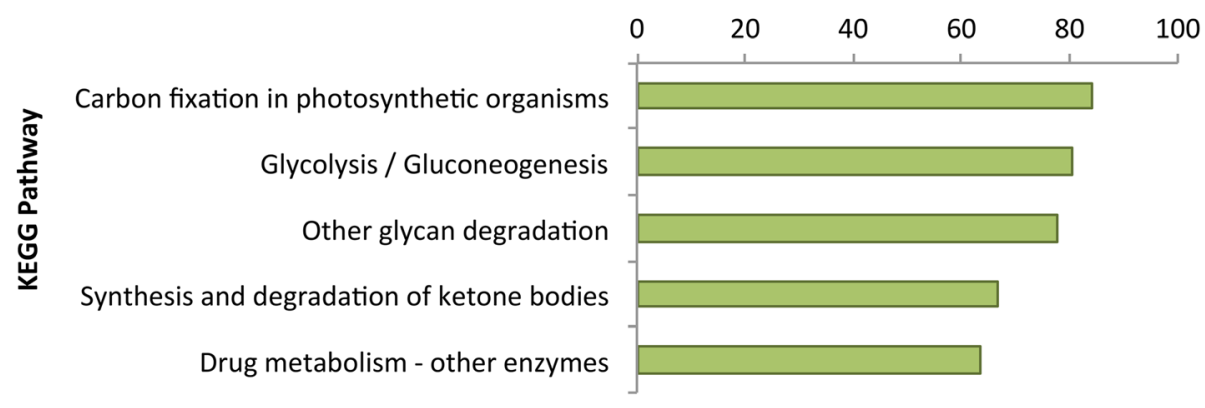

Fig. 3 Enzyme representation of the top five KEGG pathways ranked by the percentage of enzymes annotated in the pea axillary bud transcriptome

analysis between time windows 1 and 3 , while six were identified as DE in the analysis between time windows 2 and 3. The qRT-PCR results showed ten out of the 14 (71\%) transcripts were validated between time windows 1 and 3, while only two out of six (33\%) were validated between time windows 2 and 3 (Fig. 4). For the genes that were validated by qRT-PCR, very similar fold changes were identified by both the edgeR analysis and qRT-PCR (e.g., see comp 72075_c0 and comp 81803_c0 in Fig. 4). Notably, due to the short time differences, these foldchanges were generally less than 3-fold.

Only half of the total unique DE transcripts were annotated (Tables 5 and 6), which suggested an involvement of pea-specific genes, including non-coding RNA. This was confirmed as $44 \%$ of the unannotated DE transcripts were classified as putative lncRNA using our criteria (Table 5; Additional file 1: Table S19); however, none of the DE transcripts showed sequence homology to Rfam and repetitive DNA databases. This suggests that as well as affecting expression of protein-coding genes, the time of sample collection also affects expression of putative lncRNAs. As not all of the unannotated transcripts were classified as putative lncRNA, the remaining unannotated transcripts could have other functions such as encoding as yet unknown or pea-specific proteins or small RNA precursors.

lncRNAs have previously been associated with diurnal changes and light responses [32]. Hazen et al. [33] looked for non-coding sequences that exhibited rhythmic expression and identified 1052 intergenic regions of the Arabidopsis genome that had rhythmic expression. In addition, 7\% of protein-coding genes exhibited rhythmic expression of lncRNA on the antisense strand, otherwise known as natural antisense transcripts (NATs). Interestingly, they also found that a number of circadian clock genes had NATs that exhibited diurnal fluctuations. This included PSEUDORESPONSE REGULATOR (PRR5), a transcriptional repressor that regulates key clock genes [34], which was also identified as DE in our study; the transcript comp90486_c1 assembly contained six isoforms, five of which were annotated as PRR5 and one of which was annotated as a putative lncRNA. Unfortunately, as our libraries are not stranded, we were unable to confirm the putative lncRNA isoform as a bona fide NAT. We also found that ten of the DE transcripts identified in our analysis were identified by Nakamichi et al. [34] as being bound and/or upregulated by PRR5 (Additional file 1: Table S20).

\section{Enrichment analysis}

To examine the function of the DE transcripts, we performed a Fisher's Exact Test $(\mathrm{FDR}<0.05)$ to identify GO terms that were enriched in the annotated DE transcript data set compared to the reference transcriptome.

There was a significant, 9-fold enrichment in the number of DE transcripts that were annotated with the GO terms rhythmic process and circadian rhythm (Fig. 5), which reflects the diurnal changes occurring in the buds. We have identified 13 genes in this set that have been characterized in the literature as being light-regulated or circadian clock-associated (Additional file 1: Table S21). In addition, 29 (40\% of annotated transcripts) DE genes

Table 5 Differentially expressed transcripts (FDR < 0.05) between pairwise comparisons of each time window collected over a time frame of 170 min in Pisum sativum buds

\begin{tabular}{llllll}
\hline Pairwise comparison & Down & Up & Total & Annotated & Potential long non-coding RNA \\
\hline Time window 1 vs 2 & 0 & 0 & 0 & n/a & n/a \\
Time window 1 vs 3 & 58 & 66 & 124 & $62(50 \%)$ & $28(23 \%)$ \\
Time window 2 vs 3 & 5 & 32 & 37 & $13(35 \%)$ & $5(14 \%)$ \\
Unique transcripts $^{\mathrm{a}}$ & 61 & 81 & 142 & $71(50 \%)$ & $31(22 \%)$ \\
\hline
\end{tabular}

${ }^{a}$ represents unique transcripts from all three pairwise comparisons 

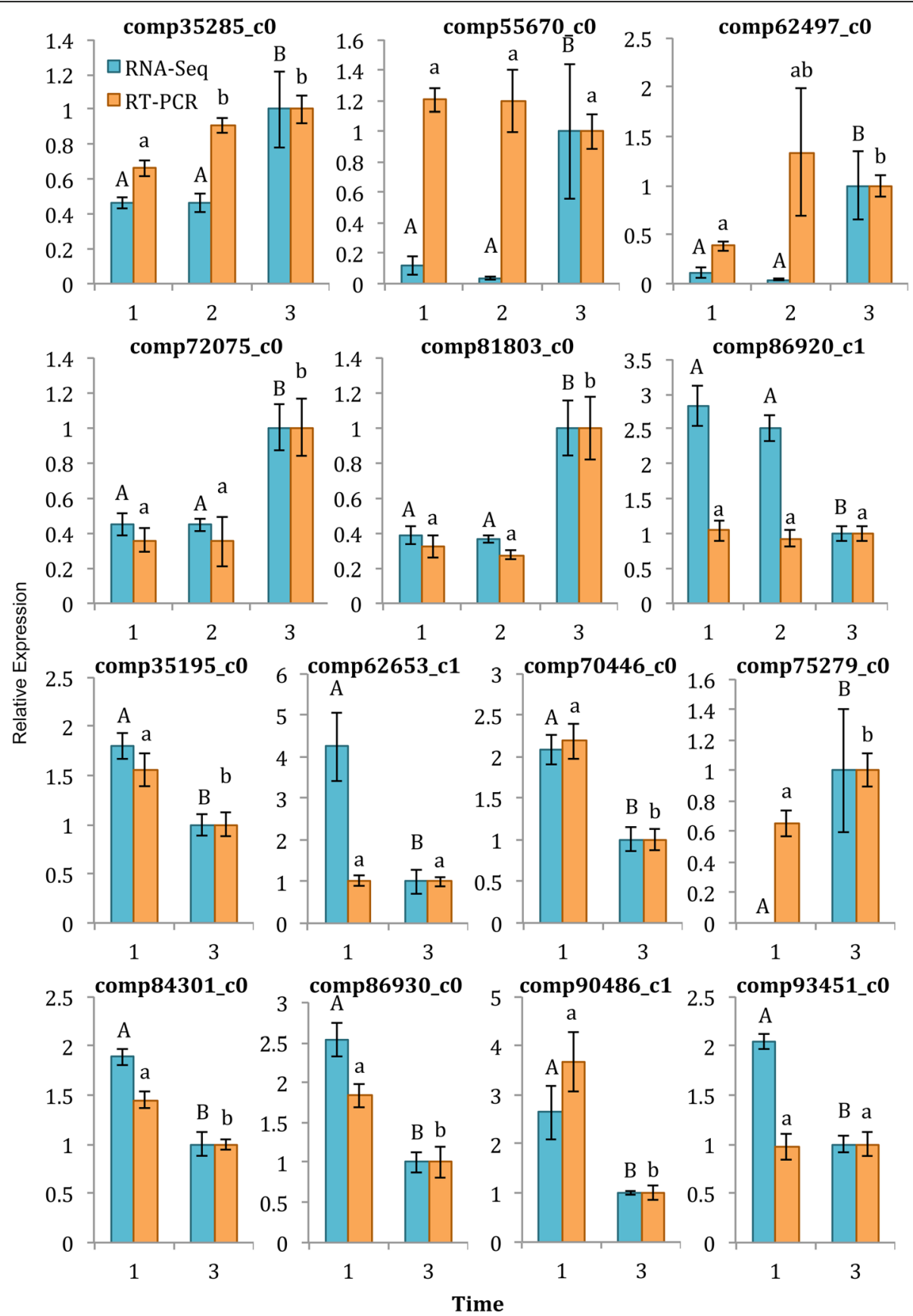

Fig. 4 Validation of 14 differentially expressed transcripts using qRT-PCR. Transcript expression in node 2 rms5-3 axillary buds was calculated relative to time window 3 for both RNA-seq data (blue bars) and qRT-PCR data (orange bars). Only time windows that were statistically significantly different in the RNA-seq edgeR analysis were included. Data are means \pm SE ( $n=3-4$ pools of $\sim 60$ plants). Statistically significant differences were determined using a one-way ANOVA with a post-hoc Tukey's test. Different letters (capital letters for RNA-seq values, lower case letters for qRT-PCR values) represent statistically significant differences at FDR $<0.05$ or $p<0.05$, respectively

identified in this study were previously identified as diurnally regulated by Blasing et al. [35] (Additional file 1: Table S22).

Importantly, we also identified six (40\%) GO terms related to post-transcriptional modifications including RNA methylation, ribonucleoprotein complex and nuclear speck (Fig. 5). This finding is consistent with previous studies showing a role of post-transcriptional regulation in diurnal gene expression changes [32, 36].

We also examined the KEGG pathways that were represented by the DE genes by comparing the percentage of annotated DE genes annotated in the each KEGG pathway 
Table 6 List of annotated differentially expressed transcripts identified in pairwise comparisons $(F D R<0.05)$ between the three time windows in Pisum sativum buds collected over a 170 min time frame

\begin{tabular}{|c|c|c|}
\hline Transcript & AT\# & Arabidopsis protein annotation \\
\hline comp103811_c0 & AT5G16080 & Probable carboxylesterase 15 \\
\hline comp34724_c0 & AT4G30110 & Cadmium/zinc-transporting ATPase HMA2 \\
\hline comp34931_c0 & AT1G15760 & Sterile alpha motif domain-containing protein \\
\hline comp35134_c1 & AT5G02570 & Histone h2b \\
\hline comp35188_c0 & AT2G43460 & 60 s ribosomal protein 138 \\
\hline comp35195_c0 & AT3G62980 & Protein TRANSPORT INHIBITOR RESPONSE 1 \\
\hline comp35285_c0 & AT1G14450 & NADH dehydrogenase \\
\hline comp35297_c0 & AT4G26470 & Calcium-binding protein CML21 \\
\hline comp35867_c0 & AT2G16365 & F-box protein \\
\hline comp36828_c0 & AT3G48770 & ATP/DNA binding protein \\
\hline comp42921_c0 & AT1G68050 & Kelch repeat-containing protein \\
\hline comp54867_c0 & AT1G02070 & Uncharacterized protein \\
\hline comp55051_c0 & AT1G27480 & Lecithin-cholesterol acyltransferase-like 1 \\
\hline comp55149_c0 & AT3G24150 & Uncharacterized protein \\
\hline comp55670_c2 & ATMG00030 & Uncharacterized mitochondrial protein ATMG00030 \\
\hline comp55866_c0 & AT4G00100 & 40 s ribosomal protein s13-2 \\
\hline comp55974_c0 & AT3G61610 & Galactose mutarotase-like superfamily protein \\
\hline comp64053_c1 & AT3G12587 & Oligosaccaryltransferase \\
\hline comp68925_c0 & AT3G55340 & Phragmoplastin interacting protein 1 \\
\hline comp69006_c0 & AT3G47570 & Receptor kinase \\
\hline comp70446_c0 & AT5G24930 & Zinc finger protein constans-like 4 \\
\hline comp70806_c0 & AT1G75540 & Constans-like b-box zinc finger protein \\
\hline comp71289_c1 & AT1G07770 & 40 s ribosomal protein s15a \\
\hline comp71932_c0 & AT5G25450 & Cytochrome bd ubiquinol oxidase \\
\hline comp72075_c0 & AT1G04400 & Cryptochrome 2 \\
\hline comp73339_c0 & AT3G11050 & Ferritin 2 \\
\hline comp75279_c0 & AT2G03340 & WRKY transcription factor 3 \\
\hline comp75525_c2 & AT2G37620 & Actin 1 \\
\hline comp77858_c0 & AT4G40030 & Histone h3 \\
\hline comp77929_c0 & AT4G29390 & 40s ribosomal protein s30 \\
\hline comp78122_c1 & AT3G54500 & Night light-inducible and clock-regulated 2 \\
\hline comp78315_c0 & AT1G78510 & Solanesyl diphosphate synthase 1 \\
\hline comp79509_c0 & AT1G69180 & Transcription factor crc \\
\hline comp79848_c1 & AT5G24780 & Acid phosphatase VSP1 \\
\hline comp80157_c2 & AT1G07050 & $\mathrm{CCT}$ motif family protein \\
\hline comp81803_c0 & AT5G42900 & Cold regulated protein 27 \\
\hline comp82468_c1 & AT2G05960 & Retroelement pol polyprotein \\
\hline comp82517_c0 & AT5G02560 & Histone h2a \\
\hline comp83232_c1 & AT3G15620 & UV repair defective 3 \\
\hline comp83562_c0 & AT4G38960 & B-box type zinc finger-containing protein \\
\hline comp83593_c0 & AT2G25530 & AFG1-like ATPase family protein \\
\hline comp83707_c0 & AT3G49430 & Ser arg-rich protein 34a \\
\hline comp84080_c0 & AT5G35970 & P-loop containing nucleoside triphosphate hydrolases superfamily protein \\
\hline
\end{tabular}


Table 6 List of annotated differentially expressed transcripts identified in pairwise comparisons (FDR < 0.05) between the three time windows in Pisum sativum buds collected over a 170 min time frame (Continued)

\begin{tabular}{|c|c|c|}
\hline comp84301_c1 & AT2G24820 & Translocon at the inner envelope membrane of chloroplasts 55 \\
\hline comp84585_c0 & AT5G58140 & Phototropin 2 \\
\hline comp85037_c0 & AT4G24290 & Mac perforin domain-containing protein \\
\hline comp85451_c1 & AT2G44740 & Preg1-like negative regulator \\
\hline comp85721_c1 & AT2G42750 & DNAJ heat shock $\mathrm{N}$-terminal domain-containing protein \\
\hline comp86930_c0 & AT2G40130 & Double clp-n motif-containing p-loop nucleoside triphosphate hydrolases superfamily protein \\
\hline comp87035_c5 & AT2G38540 & Nonspecific lipid-transfer protein \\
\hline comp87071_c2 & AT3G61130 & Galacturonosyltransferase 3 \\
\hline comp87121_c0 & AT5G66290 & Uncharacterized protein \\
\hline comp87227_c2 & AT1G18330 & MYB-related transcription factor EPR1 \\
\hline comp87716_c1 & AT3G62330 & CCHC-type zinc knuckle protein \\
\hline comp88021_c0 & AT1G56220 & Dormancy auxin associated protein \\
\hline comp89415_c1 & AT3G20810 & Jumonji-C domain-containing protein 30 \\
\hline comp89617_c0 & AT5G24850 & Cryptochrome 3 \\
\hline comp89772_c0 & AT5G56850 & Uncharacterized protein \\
\hline comp90486_c1 & AT5G24470 & Pseudo-response regulator 5 \\
\hline comp90925_c6 & AT3G19900 & Uncharacterized protein \\
\hline comp91531_c2 & AT3G14050 & RelA-SpoT like protein $\mathrm{RSH} 2$ \\
\hline comp91844_c1 & - & Hypothetical protein \\
\hline comp91844_c4 & - & Hypothetical protein \\
\hline comp92821_c0 & AT4G16146 & CAMP-regulated phosphoprotein 19-related protein \\
\hline comp93257_c0 & AT3G20390 & Endoribonuclease L-PSP family protein \\
\hline comp93445_c0 & AT5G04800 & 40s ribosomal protein $517-4$ \\
\hline comp93451_c0 & AT1G04220 & Beta-ketoacyl-synthase \\
\hline comp93952_c0 & AT3G55280 & 60 s ribosomal protein L23a-2 \\
\hline comp94434_c0 & AT3G06730 & M-type thioredoxin \\
\hline comp95443_c0 & AT5G08180 & Ribosomal protein L7ae-like \\
\hline comp99868_c0 & AT4G29040 & Regulatory particle AAA-ATPase 2A \\
\hline
\end{tabular}

with the percentage of annotated reference genes present in each KEGG pathway. We found that genes involved in the fatty acid biosynthesis pathway had the largest increase in the DE gene set, with a 9-fold change (Fig. 6). Other large increases occurred in the oxidative phosphorylation pathway (5-fold; Fig. 6).

3-KETOACYL SYNTHASE 2 (KAS2; comp93451_c0), which is involved in the first steps of very-long-chain fatty acid biosynthesis [37], and LONG-CHAIN ACYL-COA SYNTHETASE 1 (LACS1; comp87716_c1), which preferentially modifies very long chain fatty acids (VLCFAs) for wax synthesis and long-chain fatty acids for cutin synthesis [38], were both identified as DE in this study. Fatty acid synthesis occurs in the plastid to provide the components of cell membranes for all plant cells [39], and has previously been linked to diurnal changes $[40,41]$ and is also required for growth.
Increased requirements for energy needed for bud growth are met by increases in ATP levels. Oxidative phosphorylation is a key stage of respiration that occurs in the mitochondria to synthesize ATP [42]. In agreement with this, enzymes from the mitochondria respiration complex I, NADH HYDROGENASE, (comp35285_c0), and complex III, UBIQUINOL CYTOCHROME C REDUCTASE SUBUNIT 7 (QCR7; comp71932_c0), were identified as DE in this study, and could potentially be regulated in a circadian fashion, likely to regulate the balance between glycolysis, oxidative phosphorylation and photophosphorylation, as suggested by Wagner et al. [43].

\section{Conclusions}

We have presented a comprehensive pea bud transcriptome for which coding genes, putative lncRNAs, 


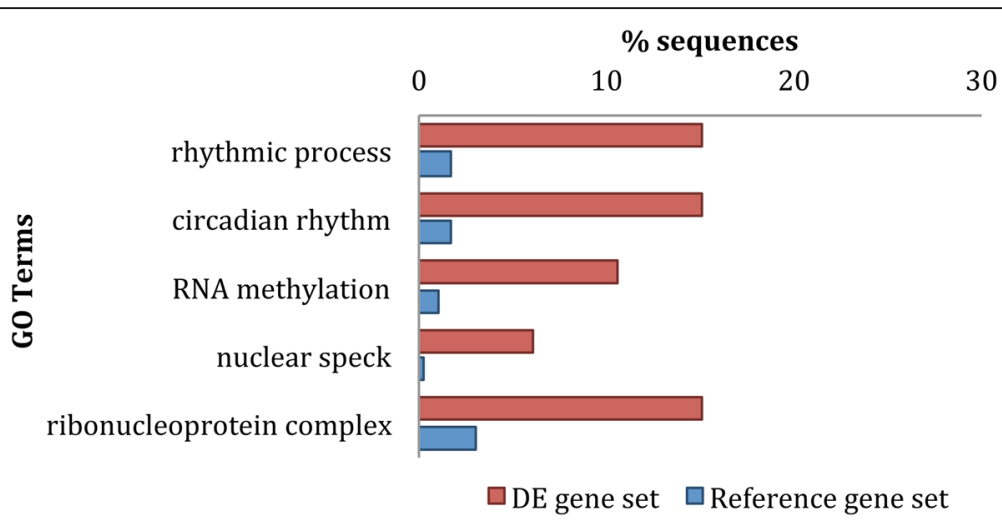

Fig. $5 \mathrm{GO}$ terms enriched in the differential expression analysis according to Fisher's Exact Test (FDR $<0.05)$, and ordered according to \% of sequences annotated in the DE gene set (red) and the reference gene set (blue). The longest isoform for each transcript in the transcriptome was used in the analysis

and miRNA precursors were identified. The assembly was validated by comparisons to other transcriptomes, which identified transcripts specific to pea axillary buds. Further analysis of this transcriptome found that growing pea buds contain many transcripts related to metabolic pathways, suggesting that the growing buds are highly active.

Gene expression was characterized in these growing axillary buds sampled in three time windows over a $170 \mathrm{~min}$ time frame. This analysis identified a number of transcripts changing over the short period of time and many of these could be annotated as genes with known or predicted diurnal regulation. It implies that changes in expression of genes known to be diurnally regulated are occurring quite rapidly in growing pea buds. The fact that we were able to discover a suite of differentially expressed genes over $170 \mathrm{~min}$, and that some of them were annotated as diurnally related, indicates that our pea bud system should be sensitive enough to identify genes that are differentially expressed in response to experimental treatments. It also implies the need for consideration of fast diurnal changes in gene expression when designing gene expression studies in pea axillary buds. Further analysis discovered that a large proportion of the DE transcripts were putative lncRNAs and coding transcripts associated with post-transcriptional modifications.

\section{Methods}

Plant growth and harvest

rms5-3 seeds were planted 4 per $2 \mathrm{~L}$ pot containing potting mix (Green Fingers B2 Potting Mix; www.greenfingerspottingmix.com). The rms5-3 (BL298) line used in this study was obtained after an initial cross between Wt15241 (rms53) and Torsdag (L107) (described in [44]), which was

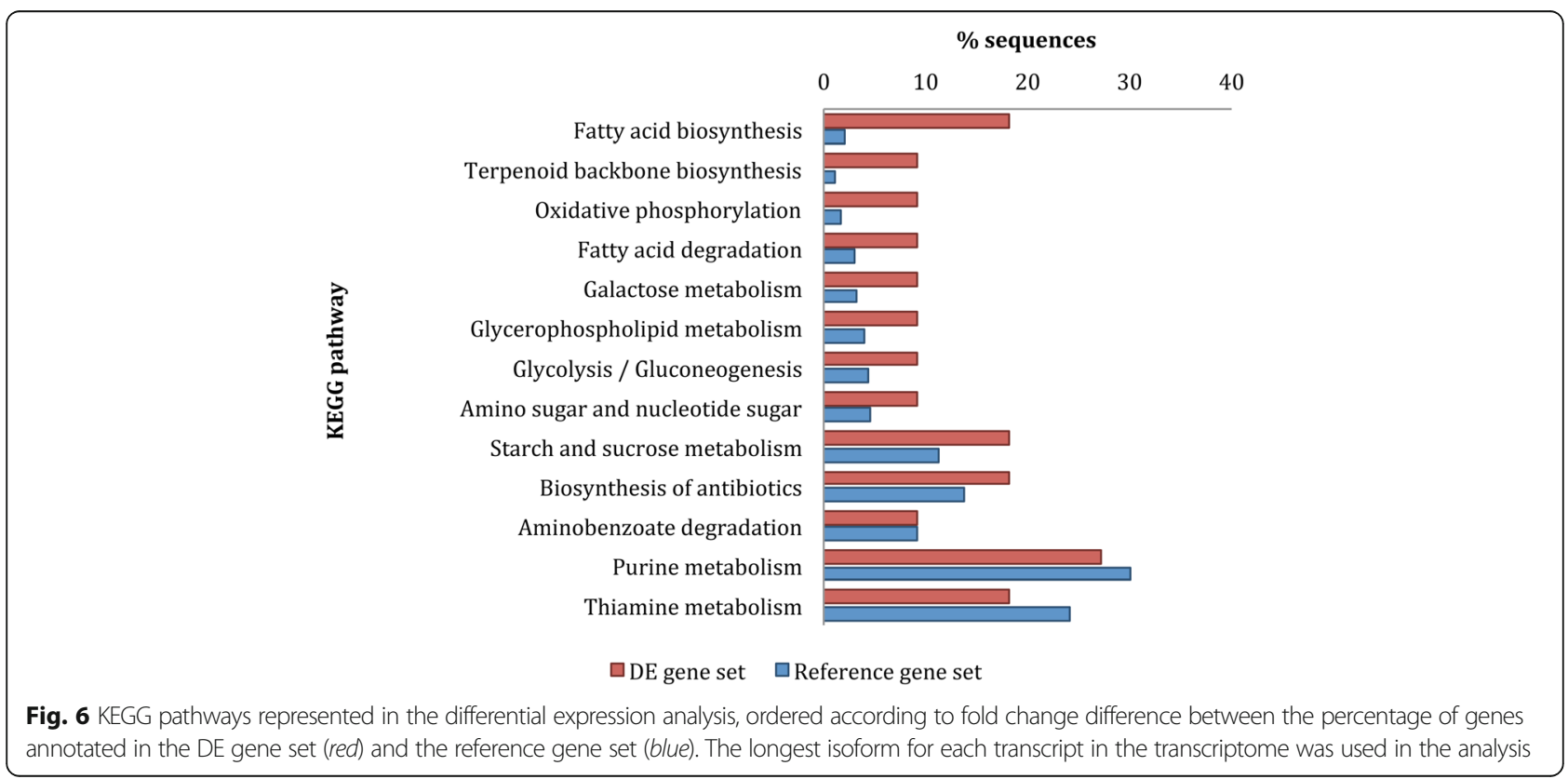


further backcrossed to Torsdag five times. Seedlings were grown in a randomized configuration until $\sim 2.5 \mathrm{LE}$, or 7 days old, under 18-h day-length glasshouse conditions as described in [45]. Node 2 buds were treated with $10 \mu \mathrm{L}$ of aqueous solution containing $0.1 \%$ Tween-20, 1\% PEG 1450, $6.25 \% \mathrm{EtOH}$ and either 0 or $1 \mu \mathrm{M}$ of the synthetic strigolactone, rac-GR24, in acetone. Node 2 buds were harvested 1, 2 , 4, or $6 \mathrm{~h}$ following treatment and immediately placed in liquid nitrogen. For the transcriptome sequencing all 24 samples were used, while for the DE analysis only the samples without the rac-GR24 treatment were used (12 oddnumbered samples 1-23 in Table S16). Approximately 3040 buds from individual plants were pooled together to make one biological replicate, with four replicates collected for each of the three treatment windows. The $\sim 840$ buds were harvested between 13:30 and 16:20 (Additional file 1: Tables S2 and S16) on the 11th October 2012 at GPS coordinates (DMS) $27^{\circ} 29^{\prime} 43^{\prime \prime} \mathrm{S}, 153^{\circ} 0^{\prime} 36^{\prime \prime} \mathrm{E}$. Those samples harvested between 1.30 and $2.10 \mathrm{pm}$ were designated as time window 1, samples harvested between 2.10 and $2.50 \mathrm{pm}$ as time window 2, and samples harvested between 3.50 and $4.30 \mathrm{pm}$ as time window 3 (Table S16).

\section{RNA extraction, library construction and sequencing}

RNA was extracted from samples using a TRIzol extraction method and then purified using RNeasy $^{\circ}$ MinElute ${ }^{\odot}$ cleanup kit (Qiagen ${ }^{\oplus}$ ). RiboZero ${ }^{\mathrm{TM}}$ Magnetic (Plant leaf) kit (Epicentre ${ }^{\oplus}$ ) was used to remove rRNA from $3.81 \mu \mathrm{g}$ of each of the samples. The removal of rRNA was confirmed using a 2100 BioAnalyser (Agilent Technologies).

The RNA libraries were prepared using the ScriptSeq ${ }^{\text {TM }}$ V2 RNA Seq Library Preparation kit (Epicentre ${ }^{\circledast}$ ), except for the following changes: the samples were incubated in the first step for $5 \mathrm{~min}$ at the lower temperature of $70{ }^{\circ} \mathrm{C}$ to reduce RNA fragmentation, the cDNA was purified using the MinElute ${ }^{\odot}$ kit (Qiagen $^{\circledR}$ ), and the ScriptSeq ${ }^{\text {TM }}$ Index primers $1,8,9,10,11$ and 12 (Epicentre ${ }^{\oplus}$ ) were used as adaptors. Strand-specificity was not utilized. Size selection was performed by running the purified libraries on an agarose gel and excising a band of RNA between 350 and 550 bp long. This was then purified using the QIAquick ${ }^{\odot}$ Gel Extraction kit (Qiagen ${ }^{\odot}$ ). The Qubit $^{\mathrm{TM}}$ dsDNA HS Assay was used to quantify the cDNA in each library.

Four libraries were pooled together for each sequencing run, with each library contributing $1.75 \mathrm{ng}$ of RNA. The pooled libraries were prepared for sequencing using a MiSeq Desktop Sequencer (Illumina ${ }^{\circ}$ ) and run individually on a 150 or 250 paired-end cycle cartridge.

\section{RNA-seq read quality control}

For each of the steps in this section, the R1 and R2 files were processed separately. FASTQC (http://www.bioinformatics.babraham.ac.uk/projects/fastqc/) was used on the reads produced by the Illumina ${ }^{\circ}$ MiSeq to assess the quality. FASTX-Toolkit version 0.0.13.2 (http://hannonlab.cshl.edu/fastx_toolkit/index.html) was used for cleaning of the reads. FASTx trimmer was used to remove 10 bases from the 5 ' ends of the reads and $\mathrm{x}$ bases from the 3 ' ends, where $\mathrm{x}$ was determined for each sample individually depending on the FASTQC results. FASTx quality trimmer was used to remove bases from the ends of reads that had a Q-score lower than 20; any reads less than 15 bp long were discarded. FASTx quality filter was used to remove reads that had more than $50 \%$ of its bases with a Q-score less than 20. FASTx clipper was used to remove any adaptor sequences from the reads; any reads less than 5 bp long were discarded. Deconseq (version 0.4.2) was run locally to remove rRNA sequences from all of the samples [46]. Finally, any unpaired reads from the R1 and R2 files were removed.

\section{De novo transcriptome assembly}

Samples 5, 8, 11, 20 and 21 were excluded from the de novo assembly due to low quality reads. The paired-end reads from the remaining samples were used in the assembly. Trinity software (version 2013-02-25) was used to assembly a de novo transcriptome from the pairedend reads with the default parameters, except a minimum k-mer coverage of 2 was specified [47, 48].

\section{Assessment of assembly \\ The pea axillary bud transcriptome redundancy and comparison to other pea gene sequences}

BLASTN (version 2.2.28+) [49] was run locally to align the isoforms in the pea bud transcriptome to each other, specifying an E-value of $1 \mathrm{E}^{-03}$ and a hit coverage of $80 \%$.

\section{Comparing transcriptomes}

BLASTN, BLASTX and TBLASTX (version 2.2.28+) [49] were run locally to align the sequences in each transcriptome (Additional file 1: Table S23) to the pea bud transcriptome, specifying an E-value of $1 \mathrm{E}^{-10}$.

\section{Swiss-Prot and UniProtKB}

BLASTX (version 2.2.28+) [49] was run locally to align UniProtKB and SwissProt databases (Additional file 1: Table S23) of pea proteins to the pea bud transcriptome, specifying an E-value of $1 \mathrm{E}^{-10}$ and a hit coverage of $80 \%$.

\section{Ultra-conserved orthologs (UCOs)}

BLASTX (version 2.2.28+) [49] was used to compare the 357 UCO protein sequences (Additional file 1: Table S23) with the pea bud transcriptome, specifying an Evalue of $1 \mathrm{E}^{-10}$ and a hit coverage of $75 \%$.

\section{Comparison to single copy sequenced genes in pea}

BLASTN (version 2.2.28+) [49] was run locally to align the single copy pea genes to the transcriptome, specifying an $\mathrm{E}$-value of $1 \mathrm{E}^{-100}$. The single copy pea genes were 
PsApxI [17], PsBRC1 [11], PsEXGT1 [19], PsFed-1 [13], PsHMG-1 [14], PsKO1 [12], PsPCNA [18], PsPETE [16], PsRMS4 [15], and PsRMS5 [15].

\section{Gene annotation and ontology}

To annotate the transcriptome, BLASTX (version 2.2.28 +) [49] was run locally to align each isoform in the transcriptome to the $\mathrm{nr}$ database of Arabidopsis thaliana proteins and the whole $\mathrm{nr}$ database, specifying an Evalue of $1 \mathrm{E}^{-10}$.

The GO and KEGG annotations were assigned by loading the Arabidopsis thaliana annotation into BLAST2GO [50] and using the mapping function to map the annotated genes.

A custom computational pipeline was used to predict long non-coding RNAs (lncRNAs). All isoforms were subjected to BLASTP 2.2.28+ (NCBI nr), BLASTX 2.2.28+ (NCBI nr), HMMER 3.0 (both Pfam-A and Pfam-B) [51], and SignalP 4.1 [52] searches. For BLASTP, HMMER, and SignalP analyses, the isoforms were translated (start to stop codon) by Getorf tool [53] and the longest unique ORF for each isoform was retained. Isoforms with an E-value less than $1 \mathrm{E}^{-04}$ in any of the search algorithms were considered protein-coding (for SignalP D-cutoff value of 0.45 was used). To reduce the number of potential spurious lncRNAs in the transcriptome, isoforms shorter than $200 \mathrm{nt}$ were removed. Any remaining isoforms of uncertain coding potential were removed by applying a strict ORF size cut-off of 50 amino acids. Finally, the Coding Potential Calculator (CPC) [54] was used to evaluate the sensitivity of our computational pipeline. Only isoforms that were classified as 'noncoding' by CPC were finally classified as putative pea bud lncRNAs. The lncRNA isoforms were compared to a collection of annotated Pisum sativum repetitive DNA sequences from Macas et al. [23] using BLAST $\left(1 \mathrm{E}^{-10}\right)$.

Infernal (version 1.1) [29] was run locally to annotate the transcriptome with Rfam, specifying an Evalue of $1 \mathrm{E}^{-02}$.

\section{Identification of differentially expressed transcripts}

Read counts for Read 1 of all mock treated samples were calculated using the default parameters for RSEM (version 2013-04-12) [55], except that the reference file was produced using no-polyA. Differential expression of transcripts between the mock treated samples at time windows 1,2 and 3 (Additional file 1: Table S16) was calculated using edgeR (Bioconductor version 3.2.4) [56]. The count tables were first filtered to remove any transcripts with less than ten read counts in total, and the library sizes were normalised. Dispersions were estimated using the Cox-Reid profile-adjusted likelihood method, and the matrix was fit to a generalized linear model (GLM). Pairwise comparisons were then made between time windows 1,2 and 3, with three samples within each time window treated as biological replicates. To determine differentially expressed genes, an FDR threshold of 0.05 was used.

\section{qRT-PCR validation}

RNA used for qRT-PCR validation was the same as was used for RNA sequencing. cDNA was synthesized from 500 ng RNA using the iScript ${ }^{\mathrm{TM}}$ reverse transcription supermix (BioRad) as per the manufacturer's instructions. The cDNA was diluted to $0.25 \mathrm{ng} / \mu \mathrm{L}$ for qRT-PCR.

qRT-PCR analyses were performed and analyzed as previously described [3]. Primer sequences were designed using Primer3 software [57] based on transcript sequences from the de novo transcriptome assembly and can be found in Additional file 1: Table S24. PsTUBULIN2 was used as the reference gene.

\section{Enrichment analysis}

BLAST2GO (version 3.0.9) [50] was used to determine enriched GO terms using a two-sided Fisher's enrichment analysis with an FDR threshold of 0.05 , and the longest isoform for each transcript was used as the reference dataset.

\section{Additional files}

\begin{abstract}
Additional file 1: Table S1. Summary of the statistics for the raw and cleaned MiSeq paired-end reads. Table S2. Read quality statistics for Read 1 and Read 2 of each of the 24 RNA libraries sequenced. Figure S1. Length distribution of the isoforms in the pea axillary bud transcriptome. Table S3. Comparison of the Pisum sativum bud transcriptome isoforms using BLASTN (1E ${ }^{-03}$ ) and specifying a minimum hit coverage of $80 \%$. Table S4. BLASTX (1E -100 results comparing single copy pea reference sequences against the pea axillary bud transcriptome. Figure S12. Length distribution of the long noncoding RNA in the pea axillary bud transcriptome. Table S8. Summary of the number of sequences with similarity to a pea repetitive DNA sequence [23] using BLAST $\left(1 \mathrm{E}^{-10}\right)$. Figure S13. The carbon fixation in photosynthetic organisms KEGG pathway with enzymes highlighted that were identified in the pea axillary bud transcriptome. Table S16. Timetable of sample harvest for the differential expression analysis. Table S17. List of 37 transcripts identified as DE in a pairwise comparison between time window 2 and time window 3. Table S18. List of 124 transcripts identified as DE in a pairwise comparison between time window 1 and time window 3 . Table S19. List of $31 \mathrm{DE}$ transcripts annotated with a long noncoding RNA. Table S20. List of DE transcripts that are bound or upregulated by PRR5 [34]. Table S21. List of DE transcripts that have previously been identified as circadian clock-associated and/or light/ diurnally-regulated [58-71]. Table S22. List of 29 DE transcripts identified as diurnally regulated in Blasing et al. [35]. Table S23. List of online resources used in this article and their sources. Table S24. List of primers used for qRT-PCR analysis. (PDF $253 \mathrm{~kb}$ )
\end{abstract}

Additional file 2: Figure S2. The number of reads aligning to the PSApxI mRNA sequence and the alignment between PsApxl mRNA and its best hit to the pea bud transcriptome, comp92406 c0 seq1. Figure S3. The number of reads aligning to the PSBRC1 CDS and the alignment between PSBRC1 CDS and its best hit to the pea bud transcriptome, comp78442_C0_seq1. Figure S4. The number of reads aligning to the PSEXGT1 mRNA and the alignment between PSEXGT1 mRNA and its best hits to the pea bud transcriptome, comp88216_c0_seq1, 5, 7, 8, 11, 13, 14. Figure S5. The number of reads aligning to the PsFed-1 CDS and the alignment between PsFed-1 CDS and its best hits to the pea bud transcriptome, comp55599_c1_seq1 and comp351273_c0_seq1. Figure S6. The number of reads aligning to the 
PSHMG-1 mRNA and the alignment between PSHMG-1 mRNA and its best hit to the pea bud transcriptome, comp81333_c0_seq1. Figure S7 The number of reads aligning to the PsKO1 mRNA and the alignment between PsKO1 mRNA and its best hits to the pea bud transcriptome, comp71699_c1_seq1, 2, 3, 4. Figure S8. The number of reads aligning to the PSPCNA mRNA and the alignment between PSPCNA mRNA and its best hit to the pea bud transcriptome, comp93178_c0_seq1. Figure S9. The number of reads aligning to the PSPETE gene and the alignment between PSPETE gene and its best hit to the pea bud transcriptome, comp92360_c0_seq1. Figure S10. The number of reads aligning to the PSRMS4 CDS and the alignment between PSRMS4 CDS and its best hit to the pea bud transcriptome, comp97254_c0_seq1. Figure S11. The number of reads aligning to the PSRMS5 CDS and the alignment between PSRMS5 CDS and its best hits to the pea bud transcriptome, comp74555_c0_seq1 and comp27382_c0_seq1. (PDF $399 \mathrm{~kb}$ )

Additional file 3: Table S5. BLAST2GO annotation of the pea axillary bud transcriptome against Arabidopsis thaliana protein sequences. (XLSX $16823 \mathrm{~kb})$

Additional file 4: Table S6. BLAST2GO annotation of the pea axillary bud transcriptome against the non-redundant (nr) protein database. (XLSX $15158 \mathrm{~kb})$

Additional file 5: Table S7. List of putative long non-coding RNA in the pea axillary bud isoform transcriptome and their open reading frame (ORF) size. Table S9. List of putative long non-coding RNA in the pea axillary bud isoform transcriptome that match to IncRNA in Medicago [21]. Table S10. List of putative long non-coding RNA in the pea axillary bud isoform transcriptome that match to NAT IncRNA in Arabidopsis [20]. Table S11. Rfam hits for pea axillary bud isoforms. Table S12. KEGG Pathway annotation of the pea axillary bud transcriptome. Table S13. Biological processes GO annotation of the pea axillary bud transcriptome. Table S14. Cellular component GO annotation of the pea axillary bud transcriptome. Table S15. Molecular function GO annotation of the pea axillary bud transcriptome. (XLSX $4242 \mathrm{~kb}$ )

\section{Abbreviations}

aa: Amino acid; ANOVA: Analysis of variance; ATP: Adenosine triphosphate; BLAST: Blast local alignment search tool; bp: Base pair; cDNA: Complementary deoxyribonucleic acid; CDS: Coding sequence; CPC: Coding potential calculator; cv: Cultivar; DE: Differentially expressed; DNA: Deoxyribonucleic acid; EtOH: Ethanol; E-value: Expect value; FDR: False discovery rate; Gb: Giga base pair; GLM: Generalized linear model; GO: Gene ontology; im-ncRNA: Intermediatesized non-coding ribonucleic acid; KEGG: Kyoto encyclopedia of genes and genomes; IncRNA: Long non-coding ribonucleic acid; LTR: Long terminal repeat; Mb: Mega base pair; miRNA: Micro ribonucleic acid; mRNA: Messenger ribonucleic acid; NADH: Nicotinamide adenine dinucleotide; NAT: Natural antisense transcript; NCBI: National Center for Biotechnology Information; ncRNA: Non-coding ribonucleic acid; ng: Nanogram; nr: Non-redundant; nt: Nucleotide; ORF: Open reading frame; PEG: Polyethylene glycol; qRTPCR: Quantitative reverse transcription polymerase chain reaction; $\mathrm{Q}^{-}$ score: Quality score; R1: Read 1; R2: Read 2; RNA: Ribonucleic acid; RNAseq: Ribonucleic acid sequencing; rRNA: Ribosomal ribonucleic acid; SE: Standard error; snoRNA: Small nucleolar ribonucleic acid; UCO: Ultraconserved ortholog; VLCFA: Very long chain fatty acid; $\mu$ L: Microliter; $\mu \mathrm{M}$ : Micromolar

\section{Acknowledgements}

The authors would like to Beveridge lab members for help with the harvest of the bud tissue for sequencing, and Selene L. Fernandez-Valverde and Tinashe G. Chabikwa for their critical comments on the manuscript.

\section{Funding}

This research was funded by The University of Queensland and Australian Research Council grant (DP110100997) and Fellowship (FT100100806) awarded to CAB, grant (DP150102086) awarded to CAB and MT, an Australian Postgraduate Award awarded to SCK, and an Australian Research Council Laureate (FL110100044) awarded to Professor Bernard Degnan under which FG was a postgraduate scholar. None of these funding sources were involved in the design of the study and collection, analysis, and interpretation of data and in writing the manuscript.

\section{Availability of data and materials}

RNA sequence data were deposited in the SRA database at NCBI with SRA Project accession number SRP068822, BioProject accession number PRJNA308776, and BioSample accession numbers SAMN04412739SAMN04412762, inclusive. The Transcriptome Shotgun Assembly project has been deposited at DDBJ/EMBL/GenBank under the accession GEZC00000000. The version described in this paper is the first version, GEZC01000000 with 71 of the 194,067 sequences further removed by NCBI due to contamination.

\section{Authors' contributions}

SCK, MT, FG and CAB designed the experiments. SCK carried out the molecular work and most bioinformatics analyses with help from MT. The long non-coding RNAs computational prediction was done by FG and MT. SCK, MT, FG and CAB prepared the manuscript. SCK, MT, FG and CAB read, commented and approved the final manuscript.

\section{Competing interests}

The authors declare that they have no competing interests.

\section{Consent for publication}

Not applicable.

\section{Ethics approval and consent to participate}

Not applicable.

\section{Received: 16 February 2016 Accepted: 9 February 2017 \\ Published online: 02 March 2017}

\section{References}

1. Teichmann T, Muhr M. Shaping plant architecture. Front Plant Sci. 2015;6:233

2. Rameau C, Bertheloot J, Leduc N, Andrieu B, Foucher F, Sakr S. Multiple pathways regulate shoot branching. Front Plant Sci. 2015:5:741.

3. Mason MG, Ross JJ, Babst BA, Wienclaw BN, Beveridge CA. Sugar demand, not auxin, is the initial regulator of apical dominance. PNAS. 2014;111(16): 6092-7.

4. Smýkal P, Aubert G, Burstin J, Coyne CJ, Ellis NTH, Flavell AJ, et al. Pea (Pisum sativum L.) in the genomic era. Agronomy. 2012;2(2):74.

5. Sudheesh S, Sawbridge TI, Cogan NOI, Kennedy P, Forster JW, Kaur S. De novo assembly and characterisation of the field pea transcriptome using RNA-Seq. BMC Genomics. 2015;16(1):611.

6. Kaur S, Pembleton L, Cogan N, Savin K, Leonforte T, Paull J, et al. Transcriptome sequencing of field pea and faba bean for discovery and validation of SSR genetic markers. BMC Genomics. 2012;13(1):104

7. Franssen SU, Shrestha RP, Brautigam A, Bornberg-Bauer E, Weber APM. Comprehensive transcriptome analysis of the highly complex Pisum sativum genome using next generation sequencing. BMC Genomics. 2011;12:227.

8. Duarte J, Riviere N, Baranger A, Aubert G, Burstin J, Cornet L, et al. Transcriptome sequencing for high throughput SNP development and genetic mapping in pea. BMC Genomics. 2014;15:126.

9. Young ND, Debellé F, Oldroyd GED, Geurts R, Cannon SB, Udvardi MK, et al. The Medicago genome provides insight into the evolution of rhizobial symbioses. Nature. 2011;480(7378):520-4.

10. Lai Z, Kane NC, Kozik A, Hodgins KA, Dlugosch KM, Barker MS, et al. Genomics of Compositae weeds: EST libraries, microarrays, and evidence of introgression. Am J Bot. 2012;99(2):209-18.

11. Braun N, de Saint Germain A, Pillot J-P, Boutet-Mercey S, Dalmais M, Antoniadi I, et al. The pea TCP transcription factor PsBRC1 acts downstream of strigolactones to control shoot branching. Plant Physiol. 2012:158(1):225-38.

12. Davidson SE, Smith JJ, Helliwell CA, Poole AT, Reid JB. The pea gene LH encodes ent-kaurene oxidase. Plant Physiol. 2004;134(3):1123-34.

13. Elliott RC, Pedersen TJ, Fristensky B, White MJ, Dickey LF, Thompson WF. Characterization of a single copy gene encoding ferredoxin I from pea. Plant Cell. 1989;1(7):681-90.

14. Gupta R, Webster Cl, Gray JC. The single-copy gene encoding high-mobilitygroup protein HMG-I/Y from pea contains a single intron and is expressed in all organs. Plant Mol Biol. 1997;35(6):987-92.

15. Johnson X, Brcich T, Dun EA, Goussot M, Haurogné K, Beveridge CA, et al. Branching genes are conserved across species. Genes controlling a novel signal in pea are coregulated by other long-distance signals. Plant Physiol. 2006;142(3):1014-26. 
16. Last DI, Gray JC. Plastocyanin is encoded by a single-copy gene in the pea haploid genome. Plant Mol Biol. 1989;12(6):655-66.

17. Mittler R, Zilinskas BA. Molecular cloning and characterization of a gene encoding pea cytosolic ascorbate peroxidase. J Biol Chem. 1992;267(30):21802-7.

18. Shimizu S, Mori H. Changes in protein interactions of cell cycle-related genes during the dormancy-to-growth transition in pea axillary buds. Plant Cell Physiol. 1998;39(10):1073-9.

19. Takano M, Fujii N, Higashitani A, Nishitani K, Hirasawa T, Takahashi H. Endoxyloglucan transferase cDNA isolated from pea roots and its fluctuating expression in hydrotropically responding roots. Plant Cell Physiol. 1999;40(2):135-42.

20. Wang H, Chung PJ, Liu J, Jang I-C, Kean MJ, Xu J, et al. Genome-wide identification of long noncoding natural antisense transcripts and their responses to light in Arabidopsis. Genome Res. 2014;24(3):444-53.

21. Wang TZ, Liu M, Zhao MG, Chen R, Zhang WH. Identification and characterization of long non-coding RNAs involved in osmotic and salt stress in Medicago truncatula using genome-wide highthroughput sequencing. BMC Plant Biol. 2015;15:131.

22. Li L, Eichten SR, Shimizu R, Petsch K, Yeh CT, Wu W, et al. Genome-wide discovery and characterization of maize long non-coding RNAs. Genome Biol. 2014;15(2):R40.

23. Macas J, Novák P, Pellicer J, Čížková J, Koblížková A, Neumann P, et al. In depth characterization of repetitive DNA in 23 plant genomes reveals sources of genome size variation in the legume tribe Fabeae. PLoS One. 2015;10(11):e0143424.

24. Liu TT, Zhu D, Chen W, Deng W, He H, Bai B, Qi Y, Chen R, Deng XW. A global identification and analysis of small nucleolar RNAs and possible intermediatesized non-coding RNAs in Oryza sativa. Mol Plant. 2013;6:830-46.

25. Wang Y, Wang X, Deng W, Fan X, Liu TT, He G, Chen R, Terzaghi W, Zhu D, Deng XW. Genomic features and regulatory roles of intermediate-sized noncoding RNAs in Arabidopsis. Mol Plant. 2014;7:514-27.

26. Bonasio R, Shiekhattar R. Regulation of transcription by long noncoding RNAs. Annu Rev Genet. 2014;48:433-55.

27. Pegueroles $\mathrm{G}$. Secondary structure impacts patterns of selection in human IncRNAs. BMC Biol. 2016;14:60.

28. Mohammadin S, Edger PP, Pires JC, Schranz ME. Positionally-conserved but sequence-diverged: identification of long non-coding RNAs in the Brassicaceae and Cleomaceae. BMC Plant Biol. 2015;15:217.

29. Nawrocki EP, Burge SW, Bateman A, Daub J, Eberhardt RY, Eddy SR, et al. Rfam 12.0: updates to the RNA families database. Nucleic Acids Res. 2015; 43(D1):D130-7.

30. Consortium TGO. Gene Ontology Consortium: going forward. Nucleic Acids Res. 2015:43(D1):D1049-56.

31. Kanehisa M, Goto S, Sato Y, Furumichi M, Tanabe M. KEGG for integration and interpretation of large-scale molecular data sets. Nucleic Acids Res. 2012;40(D1):D109-14.

32. Romanowski A, Yanovsky MJ. Circadian rhythms and post-transcriptional regulation in higher plants. Front Plant Sci. 2015;6:437.

33. Hazen SP, Naef F, Quisel T, Gendron JM, Chen H, Ecker JR, et al. Exploring the transcriptional landscape of plant circadian rhythms using genome tiling arrays. Genome Biol. 2009;10(2):R17.

34. Nakamichi N, Kiba T, Kamioka M, Suzuki T, Yamashino T, Higashiyama T, et al. Transcriptional repressor PRR5 directly regulates clock-output pathways. Proc Natl Acad Sci U S A. 2012;109(42):17123-8.

35. Bläsing $O E$, Gibon $Y$, Günther M, Höhne M, Morcuende R, Osuna D, et al. Sugars and circadian regulation make major contributions to the global regulation of diurnal gene expression in Arabidopsis. Plant Cell. 2005;17(12):3257-81.

36. Kojima S, Shingle DL, Green CB. Post-transcriptional control of circadian rhythms. J Cell Sci. 2011;124(3):311-20.

37. Lee S-B, Jung S-J, Go Y-S, Kim H-U, Kim J-K, Cho H-J, et al. Two Arabidopsis 3-ketoacyl COA synthase genes, KCS20 and KCS2/DAISY, are functionally redundant in cuticular wax and root suberin biosynthesis, but differentially controlled by osmotic stress. Plant J. 2009;60(3):462-75.

38. Lu S, Song T, Kosma DK, Parsons EP, Rowland O, Jenks MA. Arabidopsis CER8 encodes LONG-CHAIN ACYL-COA SYNTHETASE 1 (LACS1) that has overlapping functions with LACS2 in plant wax and cutin synthesis. Plant J. 2009;59(4):553-64.

39. Geigenberger $\mathrm{P}$, Kolbe $A$, Tiessen A. Redox regulation of carbon storage and partitioning in response to light and sugars. J Exp Bot. 2005;56(416):1469-79.

40. Browse J, Roughan PG, Slack CR. Light control of fatty acid synthesis and diurnal fluctuations of fatty acid composition in leaves. Biochem J. 1981; 196(1):347-54.
41. Willms JR, Salon C, Layzell DB. Evidence for light-stimulated fatty acid synthesis in soybean fruit. Plant Physiol. 1999;120(4):1117-28.

42. Saraste M. Oxidative phosphorylation at the fin de siècle. Science. 1999; 283(5407):1488-93.

43. Wagner E, Frosch S, Deitzer GF. Cycles in plants. J Interdiscipl Cycle Res. 1974:5(3-4):240-6.

44. Foo E, Bullier E, Goussot M, Foucher F, Rameau C, Beveridge CA. The branching gene RAMOSUS1 mediates interactions among two novel signals and auxin in pea. Plant Cell. 2005;17(2):464-74.

45. Ferguson BJ, Beveridge CA. Roles for auxin, cytokinin, and strigolactone in regulating shoot branching. Plant Physiol. 2009;149(4):1929-44.

46. Schmieder R, Edwards R. Fast identification and removal of sequence contamination from genomic and metagenomic datasets. PLoS One. 2011; 6(3):e17288.

47. Grabherr MG, Haas BJ, Yassour M, Levin JZ, Thompson DA, Amit I, et al. Fulllength transcriptome assembly from RNA-Seq data without a reference genome. Nature Biotechnol. 2011;29(7):644-52.

48. Haas BJ, Papanicolaou A, Yassour M, Grabherr M, Blood PD, Bowden J, et al. De novo transcript sequence reconstruction from RNA-seq using the Trinity platform for reference generation and analysis. Nature Protoc. 2013;8(8):1494-512.

49. Camacho C, Coulouris G, Avagyan V, Ma N, Papadopoulos J, Bealer K, Madden TL. BLAST+: architecture and applications. BMC Bioinformatics. 2009;10:421.

50. Conesa A, Gotz S. Blast2GO: a comprehensive suite for functional analysis in plant genomics. Int J Plant Genomics. 2008;2008:619832.

51. Finn RD, Clements J, Eddy SR. HMMER web server: interactive sequence similarity searching. Nucleic Acids Res. 2011;39:W29-37.

52. Petersen TN, Brunak S, von Heijne G, Nielson H. SignalP 4.0: discriminating signal peptides from transmembrane regions. Nat Methods. 2011;8(10):785-6.

53. Rice P, Longden I, Bleasby A. EMBOSS: The European Molecular Biology Open Software Suite. Trends Genet. 2000;16(6):276-7.

54. Kong L, Zhang Y, Ye ZQ, Liu XQ, Zhao SQ, Wei L, et al. CPC: assess the protein-coding potential of transcripts using sequence features and support vector machine. Nucleic Acids Res. 2007;35:W345-9.

55. Li B, Dewey CN. RSEM: accurate transcript quantification from RNA-Seq data with or without a reference genome. BMC Bioinformatics. 2011;12:323.

56. Robinson MD, McCarthy DJ, Smyth GK. edgeR: a Bioconductor package for differential expression analysis of digital gene expression data. Bioinformatics. 2010;26(1):139-40.

57. Untergasser A, Cutcutache I, Koressaar T, Ye J, Faircloth BC, Remm M, Rozen SG. Primer3_-new capabilities and interfaces. Nucleic Acids Res. 2012:40(15):e115.

58. Song YH, Smith RW, To BJ, Millar AJ, Imaizumi T. FKF1 conveys timing information for CONSTANS stabilization in photoperiodic flowering. Science. 2012;336(6084):1045-9.

59. Xu D, Li J, Gangappa SN, Hettiarachchi C, Lin F, Andersson MX, et al. Convergence of light and $\mathrm{ABA}$ signaling on the $\mathrm{AB} / 5$ promoter. PLoS Genet. 2014;10(2):e1004197.

60. Lin CT, Yang HY, Guo HW, Mockler T, Chen J, Cashmore AR. Enhancement of blue-light sensitivity of Arabidopsis seedlings by a blue light receptor cryptochrome 2. Proc Natl Acad Sci U S A. 1998;95(5):2686-90.

61. Toth R, Kevei E, Hall A, Millar AJ, Nagy F, Kozma-Bognar L. Circadian clockregulated expression of phytochrome and cryptochrome genes in Arabidopsis. Plant Physiol. 2001;127(4):1607-16

62. Rugnone ML, Soverna AF, Sanchez SE, Schlaen RG, Hernando CE, Seymour DK, et al. LNK genes integrate light and clock signaling networks at the core of the Arabidopsis oscillator. Proc Natl Acad Sci U S A. 2013;110(29):12120-5.

63. Li G, Siddiqui H, Teng YB, Lin RC, Wan XY, Li JG, et al. Coordinated transcriptional regulation underlying the circadian clock in Arabidopsis. Nat Cell Biol. 2011;13(5):616-U275.

64. Kleine T, Kindgren P, Benedict C, Hendrickson L, Strand A. Genome-wide gene expression analysis reveals a critical role for CRYPTOCHROME1 in the response of Arabidopsis to high irradiance. Plant Physiol. 2007;144(3):1391-406.

65. Boij P, Patel R, Garcia C, Jarvis P, Aronsson H. In vivo studies on the roles of Tic55-related proteins in chloroplast protein import in Arabidopsis thaliana. Mol Plant. 2009:2(6):1397-409.

66. Christie JM, Salomon M, Nozue K, Wada M, Briggs WR. LOV (light, oxygen, or voltage) domains of the blue-light photoreceptor phototropin (nph1): binding sites for the chromophore flavin mononucleotide. Proc Natl Acad Sci U S A. 1999:96(15):8779-83.

67. Kuno N, Moller SG, Shinomura T, Xu XM, Chua NH, Furuya M. The novel MYB protein EARLY-PHYTOCHROME-RESPONSIVE1 is a component of a slave circadian oscillator in Arabidopsis. Plant Cell. 2003;15(10):2476-88. 
68. Jones MA, Covington MF, DiTacchio L, Vollmers C, Panda S, Harmer SL. Jumonji domain protein JMJD5 functions in both the plant and human circadian systems. Proc Natl Acad Sci U S A. 2010;107(50):21623-8.

69. Pokorny R, Klar T, Hennecke U, Carell T, Batschauer A, Essen LO. Recognition and repair of UV lesions in loop structures of duplex DNA by DASH-type cryptochrome. Proc Natl Acad Sci U S A. 2008;105(52):21023-7.

70. Nakamichi N, Kita M, Ito S, Yamashino T, Mizuno T. PSEUDO-RESPONSE REGULATORS, PRR9, PRR7 and PRR5, together play essential roles close to the circadian clock of Arabidopsis thaliana. Plant Cell Physiol. 2005;46(5):686-98.

71. Mizusawa K, Masuda S, Ohta H. Expression profiling of four RelA/SpoT-like proteins, homologues of bacterial stringent factors, in Arabidopsis thaliana. Planta. 2008;228(4):553-62.

\section{Submit your next manuscript to BioMed Central and we will help you at every step:}

- We accept pre-submission inquiries

- Our selector tool helps you to find the most relevant journal

- We provide round the clock customer support

- Convenient online submission

- Thorough peer review

- Inclusion in PubMed and all major indexing services

- Maximum visibility for your research

Submit your manuscript at www.biomedcentral.com/submit 